\title{
Optimum Signal Shaping for MIMO Radar System
}

\author{
Jamal S. Rahhal ${ }^{1}$ \\ ${ }^{1}$ Electrical Engineering Department, School of Engineering, The University of Jordan, Amman, Jordan \\ Correspondence: Jamal S. Rahhal, Electrical Engineering Department, School of Engineering, The University of \\ Jordan, Amman 11942, Jordan. Tel: 962-777-483-674. E-mail: rahhal@ju.edu.jo
}

Received: September 25, 2017 Accepted: October 4, 2017 Online Published: October 8, 2017

doi:10.5539/mas.v11n11p1 URL: https://doi.org/10.5539/mas.v11n11p1

\begin{abstract}
:
Multiple Input Multiple Output (MIMO) systems showed good utilization of channel characteristics. In MIMO systems multiple signals are transmitted using multiple antennas system. This provides each receiver the whole combined signal and hence, spatial diversity gain is obtained. A MIMO radar system, transmit via its diverse antennas multiple probing signals that is used to detect targets. The receiving antennas combined together form independent units. Their signals can be processed to make use from the diversity gain and better identifies the targets. In this paper, optimal probing signals are derived and its performance is simulated. MIMO radar can transmit different orthogonal signals that lead to signal diversity. Results showed good utilization of spatial diversity and better timing detection when using the optimal signals over the conventional phased array radar.
\end{abstract}

Key words: MIMO, Radar, Target Detection, DOA, Phase Array and Channel Estimation.

\section{Introduction:}

MIMO radar systems use multiple transmitter antennas to transmit the radar pulse or continuous wave signal and multiple receiving antennas to detect the reflected signals as shown in Figure 1. The transmitters and the receivers are spread over wide area to implement independent transmitters and receivers. This provides the MIMO system better capability to isolate the reflected signals from the ground clutter (Haimovich, Blum, and Cimini, 2008) and (Chen and Vaidyanathan, 2008).From its distributed nature MIMO radar stems overcome typical problems that face conventional or array radars such as targets with low radial velocities or blind speeds (Haimovich, Blum, and Cimini, 2008).

A MIMO radar system, unlike standard phased-array radar, can transmit via its antennas multiple probing signals that may be quite different from each other. This waveform diversity enables superior capabilities compared with standard phased array radar as in (Fishler et al, 2004) and (Fuhrmann and San Antonio, 2004) and (Robey, Coutts, Weikle, McHarg, and Cuomo, 2004) and (Forsythe and Bliss, 2005) and (Xu, Li, and Stoica, 2006) and (Fishler et al, 2006) and (Va and Santamara, 2008). This waveform diversity enables the MIMO radar superiority in several fundamental aspects. The waveform diversity afforded by MIMO radar enables much improved parameter identifiability over its phased-array counterpart (Haimovich, Blum, and Cimini, 2008).

The probing signal transmitted by a MIMO radar system can be designed to a proximate a desired transmit beam pattern and also to minimize the cross correlation of the signals bounced from various targets of interest. Using this design, we can significantly improve the parameter estimation accuracy of the adaptive MIMO radar techniques. We demonstrate the advantages of these MIMO transmit beam pattern designs over their phased-array counterparts. Due to the significantly larger number of degrees of freedom of a MIMO system, we can achieve much better transmit beam patterns with a MIMO radar, under the practical uniform elemental transmit power constraint, than with its phased-array counterpart. 


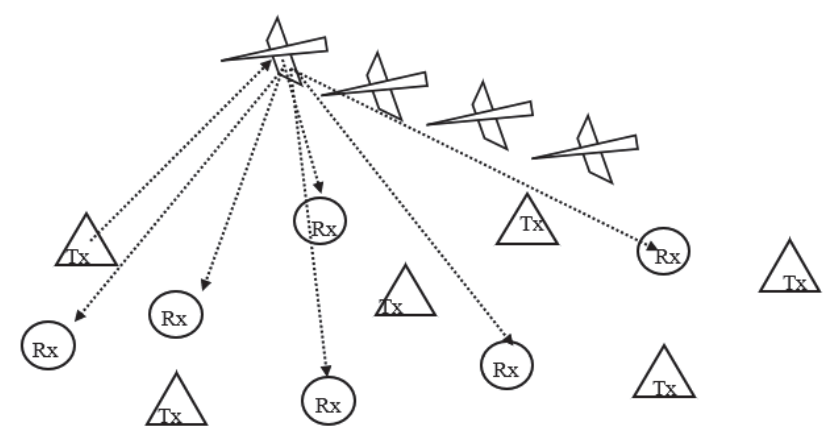

Figure 1. MIMO Radar System.

MIMO radar offers the potential for significant diversity gain as well as spatial resolution gain. Also the use of point source antennas in MIMO system simplifies the implementation of the system. In (Deng, 2012) and (WANG and LU, 2009) and (Li, Xu, and Zhang, 2017) and (Daoud, 2015) a different orthogonal waveforms were used to implement a MIMO radar system and they showed significant performance improvement in finding the Direction of Arrival (DOA) and estimation of Doppler shifts.

In the following section we derive optimal signal waveforms and use it as a pre-coding for the transmitted signals.

\section{System Description}

In this paper we devise a solution to implement a MIMO radar system by employing multiple transmitter antennas at different locations and use several receiving antennas at other locations. This will make each unit as independent receiving antenna and make use of the natural spatial separation to get diversity gain. The received signals at each unit will be sampled and sent to the main processing unit. For single target scenario the signal transmitted from the $k^{\text {th }}$ transmitter can be described as (Proakis, 2001):

$$
u_{k}(t)=\sqrt{e_{k}}\left(C_{k}^{I}(t)+j C_{k}^{I}(t)\right)
$$

where $\sqrt{e_{k}}$ is a positive gain whose value is determined according to the power control and the quadrate components $C_{k}^{I}(t)$ and $C_{k}^{Q}(t)$ are given by:

$$
C_{k}^{I}(t)=\sum_{n=1}^{N_{s}} s_{k, n}^{I} P\left(t-n T_{c}-T_{s}\right)
$$

And:

$$
C_{k}^{Q}(t)=\sum_{n=1}^{N_{s}} s_{k, n}^{Q} P\left(t-n T_{c}-T_{s}\right)
$$

where $T_{c}$ is the chip interval, $T_{s}$ is the signaling interval, $N_{s}$ the number of samples per waveform $\left(s_{k, n}^{I}, s_{k, n}^{Q}\right)$ and $P(t)$ is a root-raised cosine filter. The target reflects the signal in many directions and may cause multi-reflections in the same direction. The target effect may be seen as part of the channel effect. The scattered signal from the target is given by:

$$
x_{k}(t)=g_{k}\left(\sum_{i} C_{k}^{I}(t, i)+j \sum_{i} C_{k}^{Q}(t, i)\right)
$$

Where $g_{k}$ is an attenuation factor. The received signal at the $n^{\text {th }}$ receiver is given by:

$$
r_{n}=H_{n} x+w_{n}
$$


Where $\boldsymbol{H}_{\boldsymbol{n}}$ is the channel matrix and $\mathrm{w}$ is a complex AWGN. $\boldsymbol{H}_{\boldsymbol{n}}$ is given as:

$$
H_{n}=\left[\begin{array}{cccc}
{\left[h^{1,1}\right]} & {\left[h^{2,1}\right]} & \cdots & {\left[h^{N, 1}\right]} \\
{\left[h^{1,2}\right]} & {\left[h^{2,2}\right]} & \cdots & {\left[h^{N, 2}\right]} \\
\vdots & \vdots & & \vdots \\
& & & \\
{\left[h^{1, M}\right]} & {\left[h^{2, M}\right]} & \cdots & {\left[h^{N, M}\right]}
\end{array}\right]
$$

Where for a channel of length $L$ the convolution sub-matrices in (6) have the following structure:

$$
\left[h^{i, k}\right]=\left[\begin{array}{ccccc}
h_{o}^{i, k} & 0 & \cdots & 0 & 0 \\
h_{1}^{i, k} & h_{o}^{i, k} & & & \vdots \\
\vdots & h_{1}^{i, k} & \ddots & & 0 \\
h_{L-1}^{i, k} & \vdots & & \ddots & 0 \\
0 & h_{L-1}^{i, k} & & & h_{o}^{i, k} \\
\vdots & 0 & \ddots & & h_{1}^{i, k} \\
0 & \cdots & & \ddots & \vdots \\
& & & & h_{L-1}^{i, k}
\end{array}\right]
$$

The actual channel response for each link can be represented by two orthogonal parts (Forsythe and Bliss, 2005) and (Fishler et al, 2006): the estimated part and the estimation-error part. That is, between target $i$ and receiver $n$

$$
\underbrace{h^{i, n}}_{\text {correct }}=\underbrace{\hat{h}^{i, n}}_{\text {estimated }}+\underbrace{h_{e}^{i, n}}_{\text {error }}
$$

The effect of channel estimation error reflects itself as error in the channel matrix and it is given as:

$$
H=\hat{H}+H_{e}
$$

for non-ideal case the received signal at the $n^{\text {th }}$ antenna is:

$$
r_{n}=\hat{H} x+H_{e} x+w_{n}
$$

And:

$$
r_{n}=\underbrace{\sqrt{e} \Delta \widetilde{s}^{n}}_{\text {Desired }}+H_{e} x+w_{n}
$$

To find the optimum receiver for the case of existing residual interference due to errors in channel estimation. We express the system performance in terms of SINR, and then find the receiver that satisfies the best performance (maximum SINR). Instead of correlating the received signal by the spreading code, which is the normal case, we assume a general vector $\mathbf{b}$ of unknown weights, and find the weights that satisfy the maximum SINR.

Correlating the signal received at the $n^{\text {th }}$ terminal with vector b results in the following expression:

$$
v_{n}=\left\langle r_{n}, b_{n}\right\rangle=\underbrace{\left(\sqrt{\mathrm{e}_{\mathrm{n}}}\right) b_{n}^{H} s_{n}}_{\text {Desired }}+\underbrace{b_{n}^{H} H_{e} r_{n}+b_{n}^{H} w_{n}}_{\text {Undesired }}
$$

Where the superscript $\boldsymbol{H}$ denotes the hermitian operator. From which the SINR can be expressed as:

$$
\operatorname{SINR}_{n}=\frac{e_{n}\left\|b_{n}^{H} \cdot S_{n}\right\|}{b_{n}^{H}\left(E\left\{\left\|H_{e} \cdot r_{n}\right\|\right\}+\sigma^{2}\right) b_{n}}=\frac{e_{n}\left\|b_{n}^{H} \cdot s_{n}\right\|}{b_{n}^{H} \Psi b_{n}}
$$


Where $\Psi$ used for simplifying the notation. It is desired to find $b_{n}$ that maximizes the above expression. Maximization is performed by minimizing the denominator and reserving the desired power by the following restriction: $s_{n}^{H} b_{n}=1$.The solution comes from the generalized eigenvalue problem. Where in general for a hermitian matrix A the maximum and minimum values of the expression:

$$
z=\frac{y^{H} A y}{y^{H} y}
$$

are the maximum and minimum eigenvalues of A, respectively. Equation (14) can be written in a more general form, as:

$$
z=\frac{y^{H} A y}{y^{H} R y}
$$

Finding the minimum of this equation is equivalent to finding the general case eigenvector (Lamare and Sampaio-Neto, 2011) and (Kay, 1993):

$$
A \cdot y=\lambda_{\min } R \cdot y
$$

Applying this to (15) is equivalent to finding the minimum of its reciprocal, and hence:

$$
\Psi . b_{n}=\lambda_{\min } s_{n} \cdot s_{n}^{H} b_{n}
$$

making use of the power restriction $s_{n}^{H} b_{n}=1$ and solving for $\boldsymbol{b}$ we get:

$$
b_{n}=\mu \Psi^{-1} s_{n}
$$

Where $\mu$ is a constant, making use of the matrix inversion lemma, the above expression could be simplified to:

$$
b_{n}=\frac{\mu}{\sigma_{n}^{2}}\left[I-\frac{E\left\{\left\|H_{e} r_{n}\right\|\right\}}{\sigma_{n}^{2}+\left(\sigma_{e}^{2} M r_{n}^{H} r_{n}\right)}\right] S_{n}
$$

Note in the above expression if the channel estimation error is negligible then the above vector reduces simply to the spreading vector $S_{n}$.

The result in (19) represents the optimum receiver which shows obvious improvement in system performance especially for cases of large estimation errors, while for small estimation errors the optimum receiver returns simply to that of a single code de-spreader for which the interference from other transmitters is cancelled by the zero forcing weights of the space-time filter.

At the transmitter we pre-code the spreading sequence $\widetilde{s}_{n}$ as:

$$
\widetilde{s}_{n}=\left[\frac{\mu}{\sigma_{n}^{2}}\left[I-\frac{E\left\{\left\|H_{e} r_{n}\right\|\right\}}{\sigma_{n}^{2}+\left(\sigma_{e}^{2} M r_{n}^{H} r_{n}\right)}\right]\right]^{-1} s_{n}
$$

Therefore, at the receiver we use the regular de-spreading codes to optimally detect the transmitted signal.

In this paper we devise the use of Constrained BLUE estimator to estimate the channel matrix. To derive the constraint condition we minimize the error variance that is given by (Krieger, 2013) and (Shahbazpanahi, Gershman, and Manton, 2005) (Amayreh and Rahhal, 2004):

$$
J_{C}=(r-H x)^{T} B^{-1}(r-H x)+\lambda^{T}(A x-d)
$$


$\mathrm{B}$ is either the weight matrix or noise covariance matrix, $\mathrm{A}$ is the detection matrix and $\mathrm{d}$ is the correct data vector.Then imposing the constraint $(\mathrm{AX}=\mathrm{d})$ leads to the constrained estimate that is given by:

$$
\hat{X}_{C}=\hat{X}-\left(H^{T} B^{-1} H\right)^{-1} A^{T}\left[A\left(H^{T} B^{-1} H\right)^{-1} A^{T}\right]^{-1}(A \hat{X}-d)
$$

The received signal $\hat{X}$ represents either a noisy measurement or pre-decision for a noisy measurement of the physical quantity. $\hat{X}$ can is written as (Krieger, 2013) and (Shahbazpanahi, Gershman, and Manton, 2005) (Amayreh and Rahhal, 2004):

$$
\hat{X}_{B L U E}=\left(\hat{H}^{T} V_{z z}^{-1} \hat{H}\right)^{-1} \hat{H}^{T} V_{z z}^{-1} r
$$

Where $\boldsymbol{V}_{z z}$ is the noise covariance matrix and $\hat{H}$ is the estimated channel matrix. We can rewrite equation (22) as:

$$
\hat{X}_{C}=\hat{X}-\widetilde{H}^{-1} \Delta \hat{X}
$$

Where:

$$
\Delta \hat{X}=(A \hat{X}-d)
$$

And:

$$
\widetilde{H}^{-1}=\left(\hat{H}^{T} V_{z z}^{-1} \hat{H}\right)^{-1} A^{T}\left[A\left(\hat{H}^{T} V_{z z}^{-1} \hat{H}\right)^{-1} A^{T}\right]^{-1}
$$

If the estimated channel matrix has an estimation error, we can write it as in equation (9).This estimator will produce the channel matrix as in equation (11) and hence can be applied to determine the MIMO channel and hence, the proposed optimal waveforms can be used to detect targets in the MIMO Radar System. Each receiver will detect its own waveform and calculates the timing required to locate the target. All results are then sent to a central processor and calculate the targets locations. The error in calculating the location is reduced as the size of the system increases.

\section{Simulation and Results}

The simulation is initialized and then selects the number of codes from 255 available PN-codes. The channel matrix as generated using complex Gaussian random variable with unit variance and zero mean. The receiver's locations are generated randomly covering a wide area and one transmitter is used.

The simulation of the devised system is done for several cases using 5 targets and 4 transmitters and 32 receivers. Then it is repeated for 60 receivers. Both cases are conducted twice at 10dB SNR and at 5dB SNR.

It can be seen that the location estimate of the 5 targets is close to the true location with good resolution between close targets as shown in Figure 2 and 3.
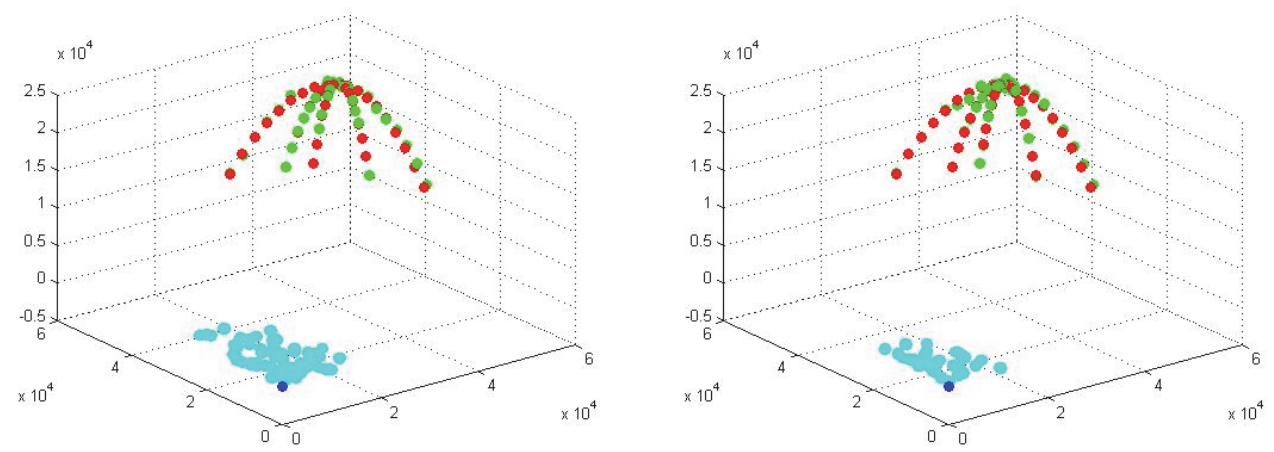

Figure 2. Five Targets using four Transmitters and 6x10 Receivers (left) and 4x8 Receivers (right) SNR=10dB 

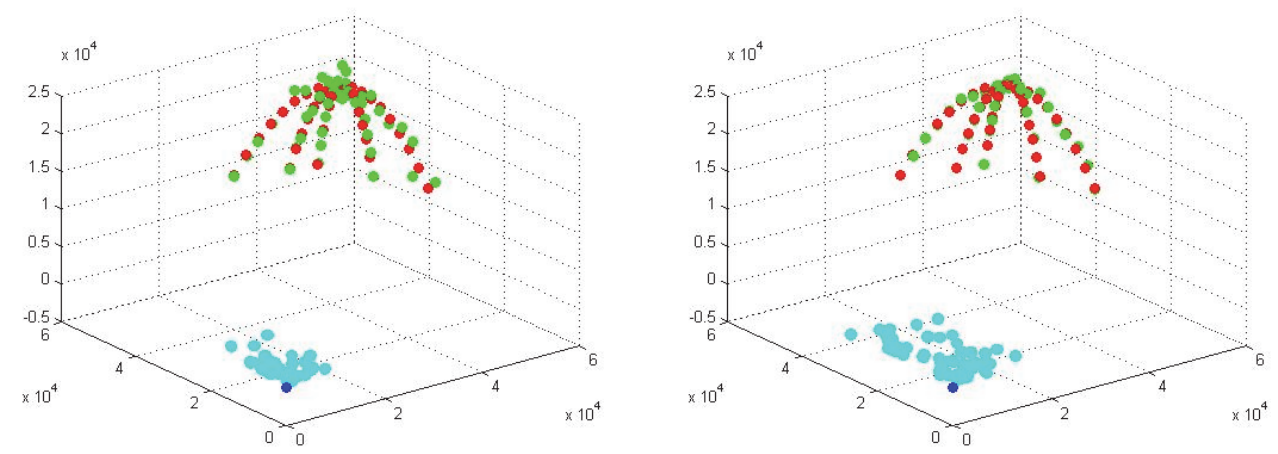

Figure 3. Five Targets using four Transmitters and 6x10 Receivers (left) and 4x8 Receivers (right) SNR=5dB.

Figure 4 shows the average percentage of error in location as a function of SNR for a 32 receivers case. Here, the decrease in error behaves exponentially as SNR increases. Figure 5 shows the average percentage of error in location as a function of number of receivers. As shown the decrease in error behaves exponentially as number of receivers increases.

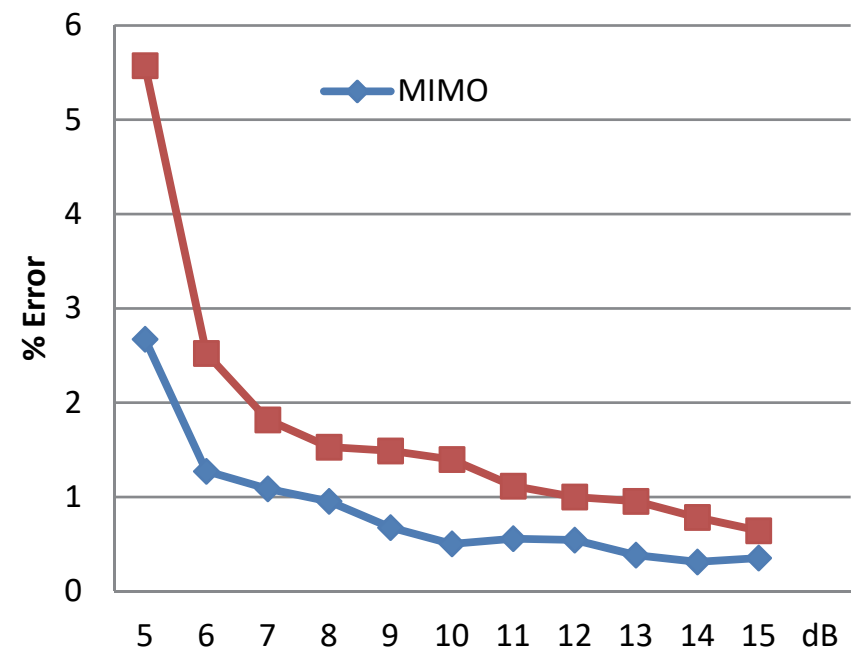

Figure 4. Percentage of Error in Location vs SNR in dB, for 32 Receivers.

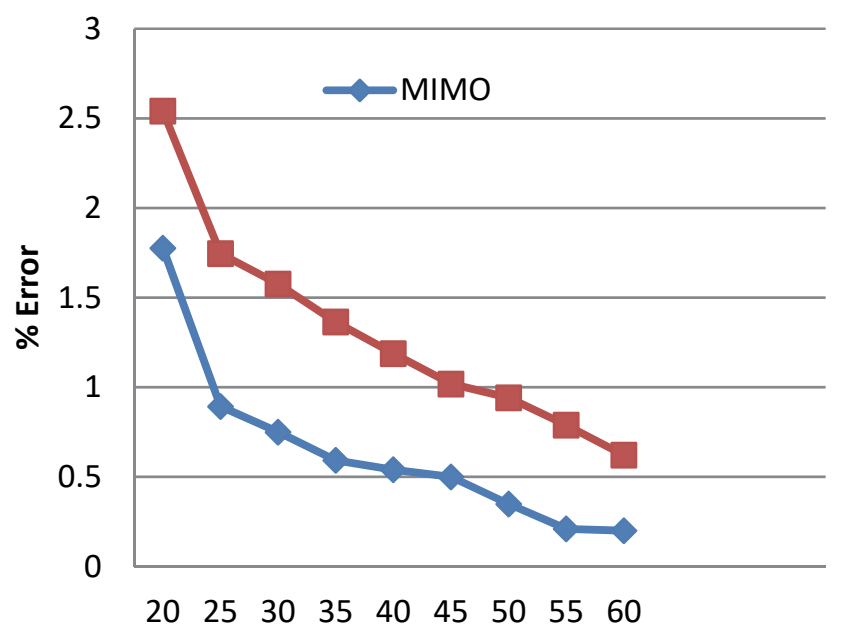

Figure 5. Percentage of Error in Location vs Number of Receivers at SNR=10dB. 


\section{Conclusion}

In this paper we derived the optimal probing waveforms for the MIMO radar system that uses orthogonal waveforms to utilize the signal diversity as well as the space diversity. Results showed a superior performance over the phased array radar. The use of optimal waveforms enhances the signal diversity and reduces the receiver complexity.

\section{References}

Alexander, M., Haimovich, R. S. B., \& Leonard, J. C. (2008). MIMO Radar with Widely Sperated Antennas. IEEE Signal Processing Magazine, 116-129. https://doi.org/10.1109/MSP.2008.4408448

Amayreh, A., \& Rahhal, J. (2004). Parallel Channels for High Rate UTRAN. International Journal of Wireless Information Networks, 11(4), 187-199. https://doi.org/10.1007/s10776-004-1311-5

Chen, C. Y., \&Vaidyanathan, P. P. (2008). MIMO Radar Space-Time Adaptive Processing Using Prolate Spheroidal Wave Functions. IEEE Transactions on Signal Processing, 56(2),623-635.https://doi.org/10.1109/TSP.2007.907917

Daoud, O. (2015). Hard Decision-Based PWM for MIMO-OFDM Radar. Communications and Network, 7 , 30-42. https://doi.org/10.4236/cn.2015.71004

de Lamare, R. C., \&Sampaio-Neto, R. (2011). Adaptive Reduced-Rank Equalization Algorithms Based on Alternating Optimization Design Techniques for MIMO Systems," IEEE Trans. Vehicular Technology, 60(6), 2482-2494. Retrieved from http://arxiv.org/abs/1301.2697v1

Deng, H. (2012). Othogonal Waveform Design for Multiple-Input Multiple-Output (MIMO) Radar. Open Journal of Applied Sciences, 2,22-25. https://doi.org/10.1007/s11767-007-0009-0

Fishler, E., Haimovich, A., Blum, R., Chizhik, D., Cimini, L., \& Valenzuela, R. (2004). MIMO radar: an idea whose time has come," Proceedings of the IEEE Radar Conference, 71-78. https://doi.org/10.1109/nrc.2004.1316398

Fishler, E., Haimovich, A., Blum, R., Cimini, L., Chizhik, D., \& Valenzuela, R. (2004). Performance of MIMO radar systems: advantages of angular diversity," 38th Asilomar Conference on Signals, Systems and Computers, Pacific Grove, CA, 1, 305-309. https://doi.org/10.1109/ACSSC.2004.1399142

Fishler, E., Haimovich, A., Blum, R., Cimini, L., Chizhik, D., \& Valenzuela, R. (2006). Spatial diversity in radars-models and detection performance. IEEE Transactions on Signal Processing, 54, 823-838. https://doi.org/10.1109/TSP.2005.862813

Forsythe, K., \& Bliss, D. (2005). Waveform correlation and optimization issues for MIMO radar. 39th Asilomar Conference on Signals, Systems and Computers, Pacific Grove, CA, 1306-1310. https://doi.org/10.1109/ACSSC.2005.1599974

Fuhrmann, D. R., \& Antonio, G. S. (2004). Transmit beamforming for MIMO radar systems using partial signal correlation," 38th Asilomar Conference on Signals, Systems and Computers, Pacific Grove, CA, 1,295-299. https://doi.org/10.1109/ACSSC.2004.1399140

Kay, S. M. (1993). Fundumentals of Statistical Signal Processing: Estimation Theory. Englewood Cliffs, NJ: Prentice-Hall.

Krieger, G. (2013). MIMO-SAR: Opportunities and pitfalls," IEEE Transactions on Geoscience and Remote Sensing, 52(5), 2628-2645. https://doi.org/10.1109/TGRS.2013.2263934

Li, H., Xu, L., \& Zhang, Z. (2017). Parameter Estimation of Maneuvering Target Using Maximum Likelihood Estimation for MIMO Radar with Colocated Antennas," Journal of Computer and Communications, 5, 69-74. https://doi.org/10.4236/jcc.2017.53008

Proakis, J. (2001). Digital Communications. $4^{\text {th }}$ edition, New York: McGraw-Hill.

Robey, F., Coutts, S., Weikle, D., McHarg, J., \& Cuomo, K. (2004). MIMO radar theory and experimental results," 38th Asilomar Conference on Signals, Systems and Computers, Pacific Grove, CA, 1, 300-304. https://doi.org/10.1109/ACSSC.2004.1399141

Shahbazpanahi, S., Gershman, A. B., \& Manton, J. H. (2005). Closed form blind mimo channel estimation for othogonal space-time codes," IEEE Transactions on Signal Processing, 53(12), 4506-4517. https://doi.org/10.1109/TSP.2005.859331

Va, J., \& Santamara, I. (2008). On the blind identifiability of orthogonal space-time block codes from second 
order statistics. IEEE Transactions on Information Theory, 54(2), 709-722.

Wang, G., \& Lu, Y. (2009). High Resolution MIMO-HFSWR Radar Using Sparse Frequency Waveforms. Wireless Sensor Network, 1(3),152-162. https://doi.org/10.1109/ICOSP.2008.4697594

Xu,L., Li, J., \& Stoica, P. (2006). Radar imaging via adaptive MIMO techniques. EUSIPCO, Florence, Italy.

\section{Copyrights}

Copyright for this article is retained by the author(s), with first publication rights granted to the journal.

This is an open-access article distributed under the terms and conditions of the Creative Commons Attribution license (http://creativecommons.org/licenses/by/4.0/). 